\title{
Transfer effects in a deductive reasoning problem
}

\author{
VICKI J. VOLBRECHT and MARIAN SCHWARTZ \\ University of Wisconsin, Madison, Wisconsin 53706
}

\begin{abstract}
Transfer in deductive reasoning was investigated through the use of isomorphic matching ("who-done-it") problems. Two groups completed three training problems followed by a test problem. For one group, the same dimensions were used in all training problems; for the other group, new dimensions were used in each training problem. The test problem involved different dimensions from any used in training. Positive transfer was found relative to a notraining control group, but the amount of transfer did not depend on whether the training had provided experience in generalizing to new dimensions. Also, time to solution did not depend on whether the problem was presented in abstract or concrete form.
\end{abstract}

The main purpose of the present experiment was to investigate positive transfer in a problem solving task involving deductive reasoning. The task employed was a variation of the type of problem that has been called the matching or "who-done-it" problem.

Transfer has been investigated in a variety of problem solving situations, including concept learning and reasoning tasks. Positive transfer has been easy to demonstrate in concept learning. For example, Bourne (1970) found large positive transfer effects when successive problems involved application of the same logical rule (conjunctive, disjunctive, conditional, or biconditional), and smaller but still significant positive transfer effects were found when successive sets of problems employed different logical rules. In an earlier study (Callantine \& Warren, 1955), performance on a test problem was measured as a function of the number of different examples of each concept used in a training series. All groups showed positive transfer on the test problem, relative to a no-training control group, and the amount of positive transfer was directly related to the number of different examples used in training. According to Callantine and Warren (1955), those subjects who had been trained on many different examples of each concept acquired a more widely generalized solution.

Positive transfer has been much harder to find in experiments using reasoning tasks. For example, Reed, Ernst, and Banerji (1974) investigated transfer between two structurally similar but not isomorphic versions of a river-crossing problem. In one experiment, no significant transfer effects were found; in another, significant positive transfer occurred only when the Jealous Husbands problem was presented before the Missionaries and Cannibals problem, and then only when the subjects were informed of the relationship between the two

Reprint requests should be sent to Marian Schwartz, Department of Psychology, W. J. Brogden Psychology Building, 1202 West Johnson Street, University of Wisconsin, Madison, Wisconsin 53706 . problems. In contrast, Luger and Bauer (1978) obtained significant positive transfer between two isomorphic problems-the Tower of Hanoi and Tea Ceremony problems-regardless of which was presented first, and even though the subjects were not informed of the relationship between the two.

The previous experiments using matching ("whodone-it") problems, by Schwartz and his co-workers, were not specifically designed to investigate transfer effects (Polich \& Schwartz, 1974; Schwartz, 1971; Schwartz \& Fattaleh, 1972). In the Schwartz (1971) experiment, an incidental finding was an absence of significant order effects over the six problems presented, but the following factors would probably have militated against order effects: (1) The form in which information was presented varied greatly from one problem to another (conjunctive, disjunctive, or conditional sentences; positive or negative sentences); and (2) subjects were able to solve only about half the problems in the time allowed, and failure to solve would have reduced transfer to subsequent problems.

The present experiment was designed to investigate transfer effects across isomorphic matching problems. Two training groups were given a series of three training problems, followed by a test problem. Subjects finished each problem before proceeding to the next. A control group was given the test problem with no prior training. The two training groups differed as follows: In the samedimensions group, the three training problems for a given subject involved the same dimensions, although different values of those dimensions; in the differentdimensions group, each training problem involved new dimensions. The test problem, which was identical for all groups, employed dimensions different from any used in the training problems. All problems used were structurally isomorphic to each other (i.e., there was a one-to-one relation between problem elements). The following predictions were made: (1) On the test problem, both training groups should perform better than the control group; that is, as the training and test problems were isomorphic, training should produce 
positive transfer (cf. Luger \& Bauer, 1978). In addition, the different-dimensions group should surpass the same-dimensions group on the test problem. The training experience of the different-dimensions group would have included the experience of generalizing the solution to new dimensions; therefore, that group should more readily generalize the solution to the new dimensions used in the test problem (cf. Callantine \& Warren, 1955). (2) Performance on the training problems should also show positive transfer. The amount of such transfer over the three training problems should reflect the amount of interproblem similarity (cf. Callantine \& Warren, 1955) and should therefore be greater in the same-dimensions than in the different-dimensions group.

A second purpose of the present experiment was to investigate the effect of the abstract-concrete variable. Previous matching-problem studies have apparently presented information only in concrete form. In studies involving conditional reasoning or syllogistic reasoning, it has generally been found that concrete material yields markedly better performance than abstract (e.g., Wason \& Johnson-Laird, 1972). In the present experiment, the test problem used abstract material, the training problems, concrete. In order to investigate the abstract-concrete variable unconfounded by order of presentation, performance on the first problem was compared for the three groups. Better performance was predicted for the two training groups, in which the first problem was concrete, than for the control group, in which the first (i.e., test) problem was abstract.

\section{METHOD}

\section{Materials}

Nine training problems and one test problem were constructed. Each problem was mimeographed on a separate page. Each problem page consisted of an introduction, followed by eight sentences, then a brief paragraph of instructions, and a table.

For the test problem, the introduction was as follows: "There are four symbols: *,,$+ \%$, and $=$. Each symbol is arbitrarily associated with a different word, and each symbol is also arbitrarily associated with a different number." The eight test-problem sentences read as follows: (1) The symbol associated with the word "unreality" is also associated with 200. (2) The symbol * is associated either with the word "indifferent" or with the word "functional." (3) The symbol $=$ is associated with 500. (4) One of the symbols is associated with the word "originate." (5) The symbol + is associated either with 500 or with 300 . (6) One of the symbols is associated with 600. (7) One of the symbols is \%. (8) The symbol associated with the word "functional" is also associated either with 200 or with 500 .

The instructional paragraph told the subjects to use the information given above to fill in the eight empty cells in the table below; that is, to enter in each row of the table the word and the number that corresponded to the symbol already entered in that same row. The table was a 3 by 4 matrix in which the three columns corresponded to the three dimensions and the four rows corresponded to the four values. The headings "Symbol," "Word," and "Number" appeared in left-to-right order over the three columns, and the four symbols were already entered in the left-hand column.
Each training problem, like the test problem, involved four values of each of three dimensions. The nine training problems comprised three versions (designated 1,2, and 3) of each of three themes (designated $\mathrm{A}$. the murder theme; $\mathrm{B}$, the picture theme; and $\mathrm{C}$, the dinner theme). The three versions of a given theme used the same dimensions but different values. For the murder theme, the three dimensions were occupation of victim, place of murder, and day of murder; for the picture theme, type of picture, name of painter, and color of frame; and for the dinner theme, main course, drink, and dessert. As an example, the introduction for Problem A1 read as follows: "Four people were victims of a murder: a judge, a farmer, an engineer, and an author. Each victim was found in a different place, and each was murdered on a different day." A sample sentence from Problem Al read: "The farmer was murdered either on Easter or on Election Day." For each problem, appropriate headings were used for the three table columns (e.g., Victim, Place, Day).

The 10 problems were isomorphic to each other. Each of the eight sentences in the test problem had its counterpart in every training problem. The counterpart presented the same information about the corresponding values of the corresponding dimensions. In order to prevent subjects from solving simply by learning position associations, the counterpart sentences appeared in a different sequence in each problem seen by a given subject. Four sequences of sentences were constructed. One sequence was used for Problems A1, B1, and C1; another for A2, $\mathrm{B} 2$, and $\mathrm{C} 2$; another for $\mathrm{A} 3, \mathrm{~B} 3$, and $\mathrm{C} 3$; and another for the test problem. The sentence sequences were random except that sequences were avoided if they appeared to make a necessary deduction unduly easy. Four different random sequences were also used for listing the four values in the left-hand column of the table.

The training problems dealt with easy-to-visualize or easy-toimagine relationships among concrete referents. The test problem dealt with arbitrary relationships among abstract words, symbols, and numbers. The four values of the word dimension (functional, indifferent, originate, and unreality) were selected to meet the following criteria: (1) They are relatively low on both the concreteness and imagery scales in the norms of Toglia and Battig (1978); (2) they bear no obvious relationship to any of the symbols or numbers used; and (3) they are relatively long. Relatively long words and three-digit numbers were used in the test problem in order to equate the amount of material (letters + digits) written by the subjects in the test problem with the mean number of letters written in the nine training problems.

\section{Subjects and Design}

The subjects were 54 student volunteers ( 40 female, 14 male) from an introductory psychology course. A block-randomization procedure was used to assign 18 subjects to each of the three groups. Exceptions to random assignment were made according to a predetermined rule in order to distribute males approximately equally over the three groups. A total of 64 subjects were run, but 10 were discarded, 4 because they failed to solve the first problem within $30 \mathrm{~min}, 5$ because they were preexperimentally familiar with the type of problem used, and 1 because of a procedural error.

Each subject in the same-dimensions group was given three training problems that represented the three versions of the same theme. Each different-dimensions subject was given three training problems that represented three different themes. Within each training group, (1) each of the nine training problems occurred twice in each of the three positions, (2) no sequence of three training problems was used by more than one subject, and (3) no subject saw the counterpart sentences in the same sequence twice. The test problem was the fourth problem given the subjects in the training groups; the control group was given the test problem without any prior training. 
Procedure

Subjects were run one at a time. The subject was seated at a table and provided with pencils and a desk bell. Preliminary oral instructions were the same for all subjects and included the following points: There would be a series of problems to solve that required reasoning but not mathematics; the exact instructions for each problem would be on the problem page; subjects should work carefully and take as much time as necessary, but not waste time; they should feel free to make any marks on the problem page; and they should ring the bell to signal completion of the problem. Speed instructions were avoided in order to discourage errors. Subjects were given only one problem page at a time.

After the subject signaled completion of a problem, the experimenter examined the eight table entries and informed the subject whether the solution was correct. If incorrect, the subject was not told which specific entry was wrong, but he was instructed to continue working on the problem until the correct solution was attained. (Only six incorrect cell entries were found altogether.) If the subject's original entries were correct, time to solution was the interval between presentation of the problem page and the subject's bell signal; otherwise, the interval between the second presentation of the problem page and the second signal was added to the time for the original (wrong) solution. Before presenting each new problem, the experimenter indicated that the instructions on the next problem page might or might not be the same as before, so they should be read carefully. The purpose of this instruction was to encourage all subjects to read all material on every page.

After completion of the last problem, subjects were asked whether they had ever done similar problems before. Those who thought they had were asked to estimate when and were asked for specific examples. The five subjects who were able to describe a similar problem they had done within the past 2 years were classified as preexperimentally familiar with the problem, and data for those subjects were discarded.

\section{RESULTS}

Mean time to solution, by problem and group, is shown in Table 1. Analysis of variance on solution times for the test problem alone showed a significant effect of groups $[F(2,51)=6.63, p<.01]$. Subsequent NewmanKeuls tests showed that, as predicted, test-problem performance for each training group significantly surpassed that for the control (ps $<.01)$. Contrary to prediction, however, there was no significant difference between the two training groups $(\mathrm{p}>.05)$.

To test for the occurrence of positive transfer over the three training problems, a 2 by 4 analysis of variance was first performed with groups (same-dimensions and different-dimensions only) as the between-subjects variable and problems (all four) as the within-subjects variable. There was a highly significant effect of problems $[F(3,102)=18.72, p<.001]$, but no significant effect of groups $(F<1)$, and no significant Problems by

Table 1

Mean Time to Solution (in Seconds)

\begin{tabular}{lcccc} 
& \multicolumn{3}{c}{ Training Problem } & \\
\cline { 2 - 4 } \multicolumn{1}{c}{ Group } & 1st & 2nd & 3rd & Prost \\
Problem \\
\hline Same-Dimensions & 449 & 284 & 258 & 253 \\
Different-Dimensions & 378 & 276 & 222 & 214 \\
Control & & & & 423 \\
\hline
\end{tabular}

Groups interaction $(F<1)$. Subsequent Newman-Keuls tests indicated that performance on the first training problem was significantly slower $(p<.01)$ than on each of the other three problems, but that the other three problems did not differ significantly among themselves $(\mathrm{p}>.05)$.

To test the effect of the abstract-concrete variable, an analysis of variance was performed that compared the three groups on the first problem only (i.e., the test problem for the control group). No significant difference was found $(F<1)$. This comparison may be biased by differential loss of subjects, however, as three control subjects and one same-dimensions subject were discarded for failing to solve the first problem within $30 \mathrm{~min}$ and were replaced by new subjects. Accordingly, a second analysis included the four original subjects, each given a time-to-solution score of $1,800 \mathrm{sec}(30 \mathrm{~min})$, rather than their replacements. After this adjustment, the mean time-to-solution scores on the first problem were 508, 378 , and $625 \mathrm{sec}$ for the same-dimensions, differentdimensions, and control groups, respectively. The outcome did not change; the analysis including the original subjects showed no significant effect of groups $[F(2,51)=1.60, p>.05]$.

Where possible, other analyses were repeated using the subjects originally discarded for failure to solve within $30 \mathrm{~min}$. The outcomes remained essentially the same.

\section{DISCUSSION}

Highly significant positive transfer was found in the present experiment; that is, training on one matching problem transferred to performance on different but structurally isomorphic problems. The effect, however, was limited largely to a reduction in time to solution between the first and second training problems; further time reductions on subsequent problems were nonsignificant. Potentially, there was substantial room for improvement after the second problem; the minimal time required for solution would appear to be the 91 or $92 \mathrm{sec}$ achieved by three subjects on the fourth (test) problem, and few other subjects approached that minimal time score, on any problem.

Contrary to predictions, no significant differences were found between the same-dimensions and different-dimensions groups. In retrospect, it appears that the use of same vs. different dimensions in the three training problems may have been a very minor manipulation in the present context. Even in the samedimensions group, there were always changes from one problem to another in the specific values used and in the sequence of sentences shown; and even in the different-dimensions group, there were salient similarities from one problem to another (e.g., in the page format, and in the required overt responses of making entries in eight table cells).

Also contrary to prediction, the use of abstract vs. concrete material had no significant effect on solution. It might be noted that this lack of effect is consistent with an interpretation of the role of concreteness in reasoning offered by Revlin, Ammerman, Petersen, and Leirer (1978) and Revlis (1975). They have suggested that concreteness facilitates reasoning only when it serves to clarify the logical meaning of the given information, for example, by blocking false conversions. In the present problems, unlike those involving conditional or syllogistic reasoning, the logical meaning of the given information seems to be as clear in the abstract as in the concrete form; 
therefore, according to the above interpretation, concrete material should confer no advantage.

\section{REFERENCES}

Bourne, L. E., JR. Knowing and using concepts. Psychological Review, 1970, 77, 546-556.

Callantine, M. R., \& Warren, J. M. Learning sets in human concept formation. Psychological Reports, 1955, 1, 363-367.

Luger, G. F., \& Bauer, M. A. Transfer effects in isomorphic problem situations. Acta Psychologica, 1978, 42, 121-131.

Polich, J. M., \& Schwartz, S. H. The effect of problem size on representation in deductive problem solving. Memory \& Cognition, 1974, 2, 683-686.

REED, S. K., ERNST, G. W., \& BanerJi, R. The role of analogy in transfer between similar problem states. Cognitive Psychology, $1974,6,436-450$.
Revlin, R., Ammerman, K., Petersen, K., \& Leirer, V. Category relations and syllogistic reasoning. Journal of Educational Psychology, 1978, 70, 613-625.

REvLIS, R. Syllogistic reasoning: Logical decisions from a complex data base. In R. Falmagne (Ed.), Reasoning: Representation and process. Hillsdale, N.J: Erlbaum, 1975.

SchwarTZ, S. H. Modes of representation and problem solving: Well evolved is half solved. Journal of Experimental Psychology, 1971, 91, 347-350.

Schwartz, S. H., \& FatTaleh, D. L. Representation in deductive problem solving. Journal of Experimental Psychology, 1972, 95, 343-348.

Toglia, M. P., \& Battig, W. F. Handbook of semantic word norms. Hillsdale, N.J: Erlbaum, 1978.

WASOn, P. C., \& Johnson-LAIRD, P. N. Psychology of reasoning: Structure and content. London: Batsford, 1972.

(Received for publication August 1, 1979.) 